\title{
INDUCCIÓN DE PARTENOCARPIA EN Opuntia spp.
}

\section{INDUCTION OF PARTHENOCARPY IN Opuntia spp.}

\author{
Óscar E. Varela-Delgadillo, Manuel Livera-Muñoz*, \\ Alfonso Muratalla-Lúa y José A. Carrillo-Salazar
}

\begin{abstract}
Posgrado en Recursos Genéticos y Productividad-Fisiología Vegetal, Campus Montecillo, Colegio de Postgraduados. km 36.5 Carr. México-Texcoco.
\end{abstract} 56230, Montecillo, Texcoco, Edo. de. México.

*Autor para correspondencia (mlivera@colpos.mx)

\section{RESUMEN}

La tuna (Opuntia spp.) es un fruto que posee cualidades apreciadas por los consumidores en México y otros países; sin embargo, algunas personas no la consumen porque tiene numerosas semillas grandes en la pulpa, lo cual afecta su calidad y limita su aceptación en los mercados. El objetivo de esta investigación fue determinar la factibilidad de producir frutos partenocárpicos de calidad en cuatro variedades de nopal tunero de amplio uso por los agricultores de Zacatecas y San Luis Potosí. Se aplicaron las dosis de 0, 50, 100 y 200 ppm de ácido giberélico $\left(\mathrm{AG}_{3}\right)$ a flores emasculadas en etapa de pre-antesis de las variedades Amarilla Montesa (Opuntia megacantha SalmDick), Burrona (Opuntia albicarpa Sheinvar), Cristalina (Opuntia albicarpa Sheinvar) y Rojo Pelón (Opuntia ficus-indica L.). A las flores testigos (0 ppm) se les dejó polinizar libremente. El diseño experimental fue completamente al azar, con 16 tratamientos resultantes de un arreglo factorial completo con dos factores y cuatro niveles para cada factor $\left(2^{4}\right)$. La unidad experimental estuvo conformada por una flor. Las aplicaciones se hicieron en 20 flores (repeticiones) de diferentes plantas por tratamiento. Se obtuvieron frutos partenocárpicos con las tres dosis de $A_{3}$, con una reducción significativa de su longitud, diámetro y peso. Los frutos con aplicación de $A G_{3}$ redujeron peso de pulpa, relación pulpa/peso de fruto y grados Brix $\left({ }^{\circ} \mathrm{Bx}\right)$ de la pulpa y de la cáscara; además se incrementó el grosor de la cáscara en comparación con los frutos provenientes de flores sin tratar (testigo). Aunque los frutos partenocárpicos contaban con poca pulpa y cáscara gruesa, esta última presentó lecturas de ${ }^{\circ} \mathrm{Bx}$ similares a las de la pulpa, por lo que la cáscara es potencialmente comestible.

Palabras clave: Opuntia, ácido giberélico, emasculación, partenocarpia, tuna.

\section{SUMMARY}

Prickly pear (Opuntia spp.) is a fruit appreciated by consumers in Mexico and other countries; however, some people do not consume it because of numerous large seeds in the pulp, which affects its quality and limits its acceptance in the markets. This research determined the feasibility of producing quality parthenocarpic fruits in four varieties of prickly pear cactus widely used by farmers of Zacatecas and San Luis Potosi. Doses of 0, 50, 100 and $200 \mathrm{ppm}$ of gibberellic acid $\left(\mathrm{GA}_{3}\right)$ were applied into emasculated flowers at the pre-anthesis stage to varieties Amarilla Montesa (Opuntia megacantha Salm-Dick), Burrona (Opuntia albicarpa Sheinvar), Cristalina (Opuntia albicarpa Sheinvar) and Rojo Pelón (Opuntia ficus-indica L.). Control flowers
(0 ppm) pollinated freely. The experimental design was completely randomized with 16 treatments resulting from a complete factorial arrangement with two factors and four levels for each factor $\left(2^{4}\right)$. The experimental unit consisted of one flower. Applications were made in 20 flowers (replications) of different plants by treatment. Parthenocarpic fruits were obtained with the three doses of $\mathrm{GA}_{3}$, with a significant reduction in their length, diameter and weight. $\mathrm{GA}_{3}$ application reduced pulp weight, pulp weight/fruit weight ratio and ${ }^{\circ}$ Brix in both pulp and peel; in addition, the peel thickness increased in comparison to fruits produced from untreated flowers (control). Although the parthenocarpic fruits had little pulp and thicker peel, the latter had ${ }^{\circ}$ Brix readings similar to those of the pulp, thus it is potentially edible.

Index words: Opuntia, gibberellic acid, emasculation, parthenocarpy, prickly pear

\section{INTRODUCCIÓN}

Los frutos del género Opuntia, llamados tunas, son apreciados por su sabor agradable y aporte de fibra, hidrocoloides (mucilagos), pigmentos (betalaínas y carotenoides), minerales (calcio y potasio) vitaminas y azúcares (Sáenz, 2004). Según ASERCA (2011), el consumo per cápita de tuna en México es de 3.9, 4.7, 4.2 y 2.1 kg en las regiones Norte, Occidente, Centro y Sur; aun con los beneficios que aporta esta fruta, algunas personas no consumen tunas por la gran cantidad de semillas en la pulpa (Corrales y Hernández, 2005). Las semillas viables son grandes y su presencia afecta en gran medida la calidad comercial y limita la aceptación de las frutas en muchos mercados debido a la cubierta dura que tienen (ElBehi et al., 2015). El tamaño promedio de las semillas es de $4.5 \mathrm{~mm}$ de largo, $3.5 \mathrm{~mm}$ de ancho y $1.6 \mathrm{~mm}$ de espesor, y su número varía de 16 a 518 semillas por fruto (Aguilar et al., 2003).

La partenocarpia es el desarrollo de un fruto sin la fertilización de los óvulos, y puede obtenerse en genotipos particulares de algunas especies en forma natural o mediante partenocarpia artificial por aplicación de varios 
productos. Desde hace décadas se ha intentado obtener frutos sin semillas, también llamados frutos partenocárpicos. (Bangerth y Schröder, 1994). En el caso del nopal, Weiss et al. (1993) reportan que el clon BS1 de Opuntia ficus-indica presenta partenocarpia vegetativa, por lo que no requiere de la polinización para el desarrollo y cuajado del fruto. Así mismo, es posible obtener frutos sin semilla con aplicaciones externas de reguladores de crecimiento a flores con diferentes métodos y épocas de aplicación; uno de los más utilizados es con dosis de ácido giberéli$\mathrm{co}\left(\mathrm{AG}_{3}\right)$, en concentraciones que van desde 50 hasta 500 ppm (De la Barrera y Nobel, 2004; Díaz y Gil, 1978; Gil et al., 1977; Gil y Espinoza, 1980; Kaaniche-Elloumi et al., 2015; Mejía y Cantwell, 2003).

En México y otros países se ha inducido la partenocarpia en nopal tunero mediante la aplicación de ácido giberélico (Corrales y Hernández, 2005; Ortiz et al., 1991); sin embargo, a la fecha no se encuentra ningún fruto partenocárpico de calidad en el mercado, lo que posiblemente se deba a que la base genética utilizada para inducir la partenocarpia ha sido estrecha, ya que se ha utilizado principalmente Opuntia ficus-indica.

México cuenta con una amplia diversidad genética de nopal tunero; a manera de ilustración, solamente en la altiplanicie meridional se han registrado 126 variedades, que corresponden a 18 especies (Reyes-Agüero et al., 2009). Los productores de nopal tunero están utilizando una amplia gama de variedades con frutos de distintos colores (blancas, amarillas, naranja, rojas y moradas, principalmente), con cualidades muy apreciadas en el mercado nacional y en el extranjero; en este último la demanda y comercialización de tunas se han incrementado sustancialmente, particularmente en los Estados Unidos de América y Canadá (Gallegos y Mondragón-Jacobo, 2011), y éstas no son de la especie $O$. ficus-indica, por lo que se considera importante investigar si algunas de ellas presentan una buena respuesta a la inducción de la partenocarpia con ácido giberélico.

El objetivo de esta investigación fue determinar la factibilidad de producir frutos partenocárpicos de calidad en cuatro variedades de amplio uso por los productores en la región de Zacatecas y San Luis Potosí, México.

\section{MATERIALES Y MÉTODOS}

\section{Sitios experimentales y material genético}

El estudio se realizó en la segunda semana de abril de 2014, directamente en huertas en producción localizadas en la comunidad de Encinitos, Pinos, Zacatecas, que se encuentra entre los paralelos $21^{\circ} 47^{\prime}$ y $22^{\circ} 45^{\prime}$ latitud $N$ y los meridianos $101^{\circ} 17^{\prime}$ y $101^{\circ}$ 50' longitud O, con altitud de $2140 \mathrm{msnm}$. Se estudiaron cuatro variedades de nopal tunero pertenecientes a tres especies: Amarilla Montesa (Opuntia megacantha Salm-Dick), Burrona (Opuntia albicarpa Sheinvar), Cristalina (Opuntia albicarpa Sheinvar) y Rojo Pelón (Opuntia ficus-indica L.) cultivadas en distintas huertas.

\section{Manejo de los lotes experimentales}

La variedad Amarilla Montesa (AM) se localiza en una huerta de 10 ha; la plantación tiene una edad de 15 años, la densidad de población es de 800 plantas ha-1 , a $2.5 \mathrm{~m}$ entre plantas y separación entre hileras de $5 \mathrm{~m}$, se ha realizado poda de cladodios y controlado malezas arbustivas y se aplicó estiércol de bovino (15 kg/planta aproximadamente) dos años antes del estudio. La variedad Rojo Pelón (RP) tiene 5 años de establecida en una superficie de 2 ha, con distancia de $5 \mathrm{~m}$ entre hileras y $4 \mathrm{~m}$ entre plantas (500 plantas/ha-1), no se han realizado podas, sólo control de arbustos y abonado con estiércol bovino un año antes del estudio. La variedad Cristalina $(\mathrm{Cr})$ está en una huerta de 1 ha, plantada a distancia de $2.5 \mathrm{~m}$ entre plantas y $4 \mathrm{~m}$ entre hileras, con una densidad de 1000 plantas $/ \mathrm{ha}^{-1}$, la edad es de 12 años, se realizaron podas moderadas y la aplicación de abono fue dos años antes del estudio. Burrona (Br) está establecida en 1 ha con densidad de 1142 plantas/ha-1 a distancia de $3.5 \mathrm{~m}$ entre hileras y $2.5 \mathrm{~m}$ entre plantas; se ha realizado poda y abonado. En ninguna de las huertas se hizo control de plagas y enfermedades.

En cada huerta se eligió una parte de la plantación de dos o tres hileras (omitiendo las de las orillas) de $60 \mathrm{~m}$ de largo aproximadamente. Se seleccionaron plantas que presentaran condiciones similares (sanas, altura, porte, edad, etc.). Se eligieron flores en etapa de pre-antesis, se emascularon y se les aplicó el $\mathrm{AG}_{3^{\prime}}$ y para el caso de las flores testigo ( 0 ppm de $\mathrm{AG}_{3}$ ) se dejó que se polinizaran libremente.

\section{Tratamientos, diseño y unidad experimental}

Se estudió el efecto del ácido giberélico $\left(\mathrm{AG}_{3}\right)$ a cuatro concentraciones: 0 (testigo), 50, 100 y 200 ppm para inducir frutos partenocárpicos en plantas de cuatro variedades de nopal tunero AM, Br, Cr y RP, lo que dio un total de 16 tratamientos. El diseño experimental fue completamente al azar, con 16 tratamientos resultantes de un arreglo factorial completo con dos factores y cuatro niveles para cada factor $\left(2^{4}\right)$. La unidad experimental estuvo conformada por una flor. Las aplicaciones se hicieron en 20 flores (repeticiones) de diferentes plantas por tratamiento. 


\section{Variables evaluadas}

Se realizaron dos cosechas, el 17 de agosto y el 14 de septiembre de 2014, cuando los frutos presentaron pigmentación en 80 \% o más de la cáscara. El corte de la tuna se hizo con navaja en la base del fruto. Los ahuates (gloquidios) de los frutos se removieron con ayuda de una escoba; en seguida se marcaron y se colocaron en cajas de cartón para su traslado al laboratorio para la toma de mediciones.

Las variables evaluadas fueron: 1) longitud de fruto (LF) en mm: se midió con un vernier digital Truper ${ }^{\circledR}$ con precisión de $0.001 \mathrm{~cm}$; 2) diámetro ecuatorial del fruto (DEF) en $\mathrm{mm}$ : con el vernier se midió la parte más ancha del fruto en su parte central; 3) grosor de cáscara (GC) en mm: se hizo un corte longitudinal al fruto, después se separó la cáscara y al centro de ésta se midió su grosor con el vernier digital; 4) peso del fruto (PF) en g y 5) peso de la cáscara (PC) en g: se obtuvieron con una balanza digital Scout $\AA^{\circledR}$ Pro con precisión de $0.01 \mathrm{~g}$; 6) peso de la pulpa (PP) en g, se obtuvo de la diferencia entre PF y PC en fresco; 7) relación peso de pulpa/peso de fruto: se obtuvo dividiendo el peso de pulpa entre peso del fruto multiplicado por 100; 8) relación de peso de cáscara/peso de fruto: se obtuvo dividiendo el peso de la cáscara entre peso del fruto multiplicado por 100; 9) grados Brix ( ${ }^{\circ} \mathrm{Bx}$ ) de la pulpa y ${ }^{\circ} \mathrm{Bx}$ de la cáscara, se extrajeron de dos a tres gotas de jugo directamente de la pulpa o de la cáscara y se colocaron en el sensor óptico de un refractómetro digital marca $A T A G O B$ PAL-1; 10) relación ${ }^{\circ} \mathrm{Bx}$ de cáscara/ ${ }^{\circ} \mathrm{Bx}$ de pulpa, se obtuvo dividiendo los ${ }^{\circ} \mathrm{Bx}$ de la cáscara entre los ${ }^{\circ} \mathrm{Bx}$ de la pulpa multiplicado por 100; 11) número de semillas o de residuos seminales (tegumentos vacíos, sin embrión) (RS), según el caso. La pulpa del fruto se colocó en una coladera de plástico y se lavó con agua a presión hasta eliminar residuos de pulpa; después, las semillas o los RS se colocaron en papel y se secaron al ambiente; 12) peso de semillas y de RS en g, se determinó en una balanza analítica Sartorius H51.

\section{Análisis estadístico}

Se realizó análisis de varianza para cada variable con el paquete SAS (Statistical Analysis System), versión 9.3. El modelo estadístico utilizado fue $Y_{i j}=\mu+V_{i}+D_{j}+V * D_{i j}+$ $E_{i j k^{\prime}}$ donde $\mu$ es la media global, $V_{i}$ el efecto del factor variedades, $D_{j}$ el efecto del factor dosis de $A G_{3^{\prime}}, V * D_{i j}$ interacción entre variedades y niveles de $A G_{3^{\prime}} E_{i j k}$ error experimental. En los casos donde se detectaron diferencias significativas entre tratamientos se aplicó la prueba de medias de Tukey con $a=0.05$.

\section{RESULTADOS Y DISCUSIÓN}

\section{Factor variedades}

Todas las variedades fueron diferentes en longitud y diámetro de fruto $(P \leq 0.05)$. Los frutos de Cristalina fueron los más largos $(90.8 \mathrm{~mm})$ y los de Burrona los más anchos (55.1 mm). Ambas variedades se caracterizan por tener el fruto de mayor tamaño dentro de las variedades comerciales, con un promedio de $9.21 \mathrm{~cm}$ de largo y 6.79 cm de ancho (Gallegos y Mondragón-Jacobo, 2011), valores similares a los encontrados en el presente estudio. Los frutos de Amarilla Montesa son un poco alargados y con diámetro angosto, mientras que los de Rojo Pelón son más pequeños (Cuadro 1). Las cuatro variedades presentaron el mismo comportamiento en peso de fruto y peso de cáscara. Los frutos de Cristalina y Burrona fueron los más pesados (137.8 y $140.8 \mathrm{~g}$, respectivamente) y con peso de cáscara similar. Los de Amarilla Montesa y Rojo Pelón tuvieron valores más bajos de peso de fruto (112.7 y 111.9 g respectivamente) y peso de cáscara (73.0 y 72.3 g, respectivamente). Cristalina resultó con cáscara gruesa (6.7 $\mathrm{mm}$ ) y por consiguiente mayor proporción del peso del fruto $(72 \%)$. En Burrona la cáscara fue más delgada $(6.0 \mathrm{~mm})$ y presentó la relación peso de cáscara/peso de fruto más baja (67\%), mientras que Rojo Pelón tuvo la cáscara más gruesa (7.2 mm); sin embargo, su proporción con respecto al fruto (69 \%) fue semejante al de las otras variedades; lo mismo sucedió en Amarilla Montesa, aunque ésta tuvo un grosor de cáscara menor (6.8 mm) (Cuadro 1).

El peso de la pulpa de las tunas de las cuatro variedades representó en promedio $30 \%$ del peso del fruto y fue Burrona la que presentó mayor proporción de pulpa (33 \%) y Amarilla Montesa la menor (29 \%). El contenido de sólidos solubles totales en Grados Brix ( ${ }^{\circ} \mathrm{Bx}$ ) fue similar en todas las variedades, alrededor de 11 para la pulpa del fruto y de 9 a 10 en la cáscara; es decir, hubo aproximadamente $1.0^{\circ} \mathrm{Bx}$ de diferencia entre la pulpa y la cáscara.

Hubo diferencia significativa entre Cristalina y Burrona en los ${ }^{\circ} \mathrm{Bx}$ de la pulpa; Cristalina tuvo el valor más alto (12 ${ }^{\circ} \mathrm{Bx}$ ) y Burrona el más bajo (11 ${ }^{\circ} \mathrm{Bx}$ ). De las cuatro variedades, Amarilla Montesa presentó mayor lectura de ${ }^{\circ} \mathrm{Bx}$ en la cáscara $\left(10.5^{\circ} \mathrm{Bx}\right)$ con sólo $8 \%$ menos que su respectiva pulpa, mientras que en las otras tres variedades fue cercano a $16 \%$ menos que en la pulpa (Cuadro 2).

\section{Factor dosis de $\mathrm{AG}_{3}$}

Se presentaron efectos de $\mathrm{AG}_{3}$ en todas las dosis aplicadas (50, 100 y 200 ppm) en las flores emasculadas, lo que produjo frutos partenocárpicos, y redujo significativamente 
Cuadro 1. Efecto promedio de la variedad de nopal sobre características del fruto.

\begin{tabular}{lccccccccc}
\hline \multirow{2}{*}{ Variedad } & \multicolumn{2}{c}{ Fruto $(\mathrm{mm})$} & \multirow{2}{*}{ GC $(\mathrm{mm})$} & \multicolumn{3}{c}{ Peso $(\mathrm{g})$} & & \multicolumn{2}{c}{ Relación (\%) } \\
\cline { 2 - 3 } & Longitud & Diámetro & & Fruto & Cáscara & Pulpa & & Pulpa/ PTF & Cáscara/PTF \\
\hline Cristalina & $90.8 \mathrm{a}$ & $53.4 \mathrm{~b}$ & $6.7 \mathrm{~b}$ & $137.8 \mathrm{a}$ & $89.2 \mathrm{a}$ & $49.1 \mathrm{a}$ & & $27.8 \mathrm{c}$ & $72.1 \mathrm{a}$ \\
Burrona & $87.4 \mathrm{~b}$ & $55.1 \mathrm{a}$ & $6.0 \mathrm{c}$ & $140.8 \mathrm{a}$ & $86.0 \mathrm{a}$ & $54.7 \mathrm{a}$ & & $33.0 \mathrm{a}$ & $66.9 \mathrm{c}$ \\
Amarilla M. & $81.5 \mathrm{c}$ & $49.0 \mathrm{~d}$ & $6.8 \mathrm{ab}$ & $112.7 \mathrm{~b}$ & $73.0 \mathrm{~b}$ & $39.7 \mathrm{~b}$ & & $29.5 \mathrm{bc}$ & $70.4 \mathrm{ab}$ \\
Rojo P. & $75.5 \mathrm{~d}$ & $51.4 \mathrm{c}$ & $7.2 \mathrm{a}$ & $111.9 \mathrm{~b}$ & $72.3 \mathrm{~b}$ & $39.2 \mathrm{~b}$ & & $31.1 \mathrm{ab}$ & $68.8 \mathrm{bc}$ \\
DSH & 3.1 & 1.5 & 0.4 & 9.0 & 5.8 & 5.4 & & 1.3 & 1.3 \\
\hline
\end{tabular}

Medias con letras iguales en las columnas no son estadísticamente diferentes (Tukey, 0.05); DSH: diferencia significativa honesta; GC: grosor de cáscara; PTF: peso total del fruto.

Cuadro 2. Efecto promedio de la variedad sobre ${ }^{\circ B}$ rix en pulpa y cáscara, y sobre el número de semillas y residuos seminales (RS).

\begin{tabular}{|c|c|c|c|c|c|}
\hline \multirow{2}{*}{ Variedad } & \multicolumn{2}{|c|}{ Grados Brix ( $\left.{ }^{\circ} \mathrm{Bx}\right)$} & \multirow{2}{*}{$\begin{array}{l}\text { Rel. }{ }^{\circ} \mathrm{Bx} \text {-Cáscara } \\
{ }^{\circ} \mathrm{Bx}-\mathrm{Pulpa}(\%)\end{array}$} & \multirow{2}{*}{ NTS y NTRS } & \multirow{2}{*}{ PTS y PTRS (g) } \\
\hline & Pulpa & Cáscara & & & \\
\hline Cristalina & $11.8 \mathrm{a}$ & $9.7 b$ & $83.7 \mathrm{~b}$ & 382.7 a & $1.8 b$ \\
\hline Burrona & $11.1 \mathrm{~b}$ & $9.2 \mathrm{c}$ & $83.3 b$ & $295.5 b$ & $2.2 \mathrm{a}$ \\
\hline Amarilla & $11.5 a b$ & $10.5 \mathrm{a}$ & $92.4 \mathrm{a}$ & $269.2 \mathrm{c}$ & $1.2 \mathrm{C}$ \\
\hline Rojo Pelón & $11.3 a b$ & 9.4 bc & $84.1 \mathrm{~b}$ & 289.5 bc & $1.2 \mathrm{c}$ \\
\hline DSH & 0.5 & 0.5 & 2.3 & 25.1 & 0.2 \\
\hline
\end{tabular}

Medias con letras iguales en las columnas no son estadísticamente diferentes (Tukey, 0.05); DSH: diferencia significativa honesta; NTS: número total de semillas; PTS: peso total de semillas; NTRS: número de residuos seminales; PTRS: peso de residuos seminales.

la longitud, diámetro y peso de fruto, peso de pulpa, relación pulpa/peso del fruto y ${ }^{\circ} \mathrm{Bx}$ en la pulpa y en la cáscara, en comparación con los frutos provenientes de flores sin emascular y sin tratar (testigo). La cáscara de los frutos tratados aumentó su grosor y peso y, en consecuencia, la relación peso de cáscara/peso de fruto fue relativamente alta y la relación peso de pulpa/peso de fruto fue baja (Cuadro 3 ), resultados que concuerdan con los obtenidos por Corrales y Hernández (2005).

La longitud y diámetro de los frutos sin tratar (testigos) fueron mayores, en un poco más de $10 \mathrm{~mm}$, con respecto a los de frutos tratados. Se obtuvieron frutos de peso de $189 \mathrm{~g}$ cuando no se aplicó $\mathrm{AG}_{3^{\prime}}$ y cuando se aplicó a la concentración de 200 ppm el peso disminuyó hasta 116 g, siendo en esta dosis donde se encontraron los frutos más pesados con respecto a las otras (50 y 100 ppm de $A G_{3}$ ).

Las concentraciones más altas de $\mathrm{AG}_{3}$ en flores emasculadas favorecieron el desarrollo de los frutos sin semilla; similarmente, Kaaniche-Elloumi et al. (2015) encontraron que una dosis de 500 ppm produce mejores resultados que una de 100 ppm, pero con frutos más pequeños que los de flores testigo polinizadas.

Con respecto a los ${ }^{\circ} \mathrm{Bx}$ del fruto tanto en pulpa como en cáscara, las aplicaciones de $\mathrm{AG}_{3}$ produjeron frutos con aproximadamente $1.0^{\circ} \mathrm{Bx}$ menos en comparación con el testigo, y no se observaron diferencias importantes entre las tres dosis empleadas (Figuras 1A y B). Resultados similares se han obtenido con el uso de varios reguladores (Aguilar et al., 2003; Ortiz et al., 1991); donde los ${ }^{\circ} \mathrm{Bx}$ en la cáscara fueron menores que en la pulpa, de 1 a 2 grados de diferencia. Los resultados indican que, durante la maduración, tanto la cáscara como la pulpa acumulan sólidos solubles totales, siendo menor la acumulación en frutos tratados con reguladores con respecto a los no tratados; en otros casos, esta variable no se afectó y la acumulación fue similar (Ortiz et al., 1991). Muratalla et al. (2002, Com. Pers. $)^{1}$ han señalado que una característica deseable en tunas partenocárpicas es que la cáscara sea dulce y comestible.

'Muratalla L. A., M. Livera M., C. Hernández R. y V. A. González H. (2002) Tuna fresa sin semilla: reseña de un nuevo concepto de calidad de fruto de nopal (Opuntia ficus-indica) hecho realidad. In: Memorias del XIX Congreso Nacional de Fitogenética. 1-5 de septiembre. Saltillo, Coahuila, México. p: 259. 
Cuadro 3. Efecto de tres concentraciones de $\mathrm{AG}_{3}$ sobre características de calidad de los frutos de cuatro variedades de nopal tunero.

\begin{tabular}{|c|c|c|c|c|c|c|c|c|}
\hline \multirow{2}{*}{ ppm de $\mathrm{AG}_{3}$} & \multicolumn{2}{|c|}{ Fruto (mm) } & \multirow{2}{*}{$\mathrm{GC}(\mathrm{mm})$} & \multicolumn{3}{|c|}{ Peso (g) } & \multicolumn{2}{|c|}{ Relación (\%) } \\
\hline & Longitud & Diámetro & & Fruto & Cáscara & Pulpa & Pulpa/PTF & Cáscara/PTF \\
\hline 0 & 93.9 a & $62.3 \mathrm{a}$ & $4.3 \mathrm{c}$ & $189.3 \mathrm{a}$ & $77.4 \mathrm{~b}$ & $112.9 \mathrm{a}$ & $58.6 \mathrm{a}$ & $41.3 \mathrm{~b}$ \\
\hline 200 & $82.8 b$ & $50.9 b$ & $7.9 \mathrm{a}$ & $116.7 b$ & 89.3 a & $27.4 b$ & $22.7 \mathrm{~b}$ & $77.2 \mathrm{a}$ \\
\hline 100 & $81.1 \mathrm{~b}$ & $49.1 \mathrm{c}$ & $7.4 \mathrm{~b}$ & $106.2 \mathrm{c}$ & $82.9 b$ & $23.3 \mathrm{bc}$ & $21.5 b$ & $78.4 \mathrm{a}$ \\
\hline 50 & $77.4 \mathrm{c}$ & $46.5 d$ & $7.1 \mathrm{~b}$ & $91.0 \mathrm{~d}$ & $70.9 \mathrm{c}$ & 19.7 C & $21.5 b$ & $78.4 \mathrm{a}$ \\
\hline DSH & 3.1 & 1.5 & 0.4 & 9.0 & 5.8 & 5.9 & 1.3 & 1.3 \\
\hline
\end{tabular}

Medias con letras iguales en las columnas no son estadísticamente diferentes (Tukey, 0.05); DSH: diferencia significativa honesta; GC: grosor de cáscara; PTF: peso total del fruto.

Cuadro 3. Continuación.

\begin{tabular}{|c|c|c|c|c|c|}
\hline \multirow{2}{*}{ ppm de $\mathrm{AG}_{3}$} & \multicolumn{2}{|c|}{ Grados Brix ( $\left.{ }^{\circ} \mathrm{Bx}\right)$} & \multirow{2}{*}{$\begin{array}{c}\text { Rel. }{ }^{\circ B} \text {-Cáscara } \\
\text { /Bx-Pulpa }\end{array}$} & \multirow{2}{*}{ NTS y NTRS ${ }^{+}$} & \multirow{2}{*}{ PTS y PTRS ${ }^{++}(\mathrm{g}$} \\
\hline & Pulpa & Cáscara & & & \\
\hline 0 & $12.4 \mathrm{a}$ & $10.4 a$ & 88.1 a & 299.7 a & $6.34 \mathrm{a}$ \\
\hline 50 & $10.9 b$ & $9.2 b$ & $85.8 \mathrm{ab}$ & $310.5 \mathrm{a}$ & $0.08 b$ \\
\hline 100 & $11.1 \mathrm{~b}$ & $9.3 b$ & $84.6 \mathrm{~b}$ & $313.8 \mathrm{a}$ & $0.09 \mathrm{~b}$ \\
\hline 200 & $11.3 b$ & $9.6 b$ & $86.0 \mathrm{ab}$ & $312.8 \mathrm{a}$ & $0.13 b$ \\
\hline DSH & 0.5 & 0.5 & 2.3 & 25.1 & 0.21 \\
\hline
\end{tabular}

Medias con letras iguales en las columnas no son estadísticamente diferentes (Tukey, 0.05); DSH: diferencia significativa honesta; NTS: número total de semillas; PTS: peso total de semillas; NTRS: número de residuos seminales; PTRS: peso de residuos seminales; ${ }^{\dagger}$ NTS y PTS hacen referencia a

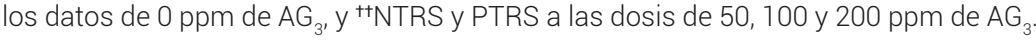

\section{Interacción variedades $\times$ concentraciones de $\mathrm{AG}_{3}$}

Las cuatro variedades presentaron la tendencia de incrementar el tamaño y peso de los frutos de flores tratadas con $\mathrm{AG}_{3}$ conforme se incrementó la dosis aplicada (Figuras $1 \mathrm{~A}, \mathrm{~B}$ y C); Cristalina y Burrona tuvieron los mayores valores. Los frutos de los testigos alcanzaron valores significativamente más altos en estas variables.

La aplicación de $\mathrm{AG}_{3}$ aumentó significativamente el grosor de la cáscara en todas las variedades y Amarilla Montesa tuvo la cáscara más delgada con las tres dosis de $\mathrm{AG}_{3}$ (Figura 1F). En Amarilla Montesa y Cristalina la dosis de 200 ppm de $\mathrm{AG}_{3}$ aumentó el peso de cáscara, encontrándose diferencias significativas con respecto al testigo y las otras dosis, excepto la de 100 ppm en Cristalina (Figura 1E). Corrales y Hernández (2005) estudiaron la calidad de tunas normales y partenocárpicas de seis variedades; la inducción de la partenocarpia la realizaron emasculando las flores y aplicando ácido indolbutírico (300 $\mathrm{mg} \mathrm{L}^{-1}$ ) y $\mathrm{AG}_{3}\left(150 \mathrm{mg} \mathrm{L}^{-1}\right.$ ) y encontraron que las variedades tuvieron comportamientos diferentes; las tunas sin semilla presentaron menor peso de fruto que los testigos y los más pesados fueron los de Cristalina. Los mismos autores encontraron que el $\mathrm{AG}_{3}$ causó alargamiento del fruto en dos variedades, efecto no encontrado en este estudio, y las tunas sin semilla presentaron mayor grosor de cáscara en casi todas las variedades, excepto en Amarilla Milpa Alta; lo que difiere de lo encontrado en esta investigación, donde el $\mathrm{AG}_{3}$ provocó el mismo efecto en las cuatro variedades estudiadas.

La emasculación impidió la formación de las semillas y el $\mathrm{AG}_{3}$ aumentó el grosor de cáscara e indujo la formación de poca pulpa en las cuatro variedades (Figura 1D). El peso de pulpa de los frutos tratados en todas las variedades no fue mayor de $35 \mathrm{~g}$, tendencia similar entre las dosis pues ninguna de ellas logró aumentar el contenido de pulpa. En contraste, la pulpa de los testigos superó los $88 \mathrm{~g}$.

En las cuatro variedades la relación pulpa/peso de los frutos partenocárpicos no alcanzó más de $26 \%$ y en los testigos ésta fue arriba de $55 \%$; sólo en Cristalina la relación peso de pulpa/peso de fruto aumentó ligeramente cuando pasó de 50 a 200 ppm y la relación peso cásca$\mathrm{ra} /$ peso de fruto disminuyó, lo cual es un efecto deseable (Figuras 2A y B). 

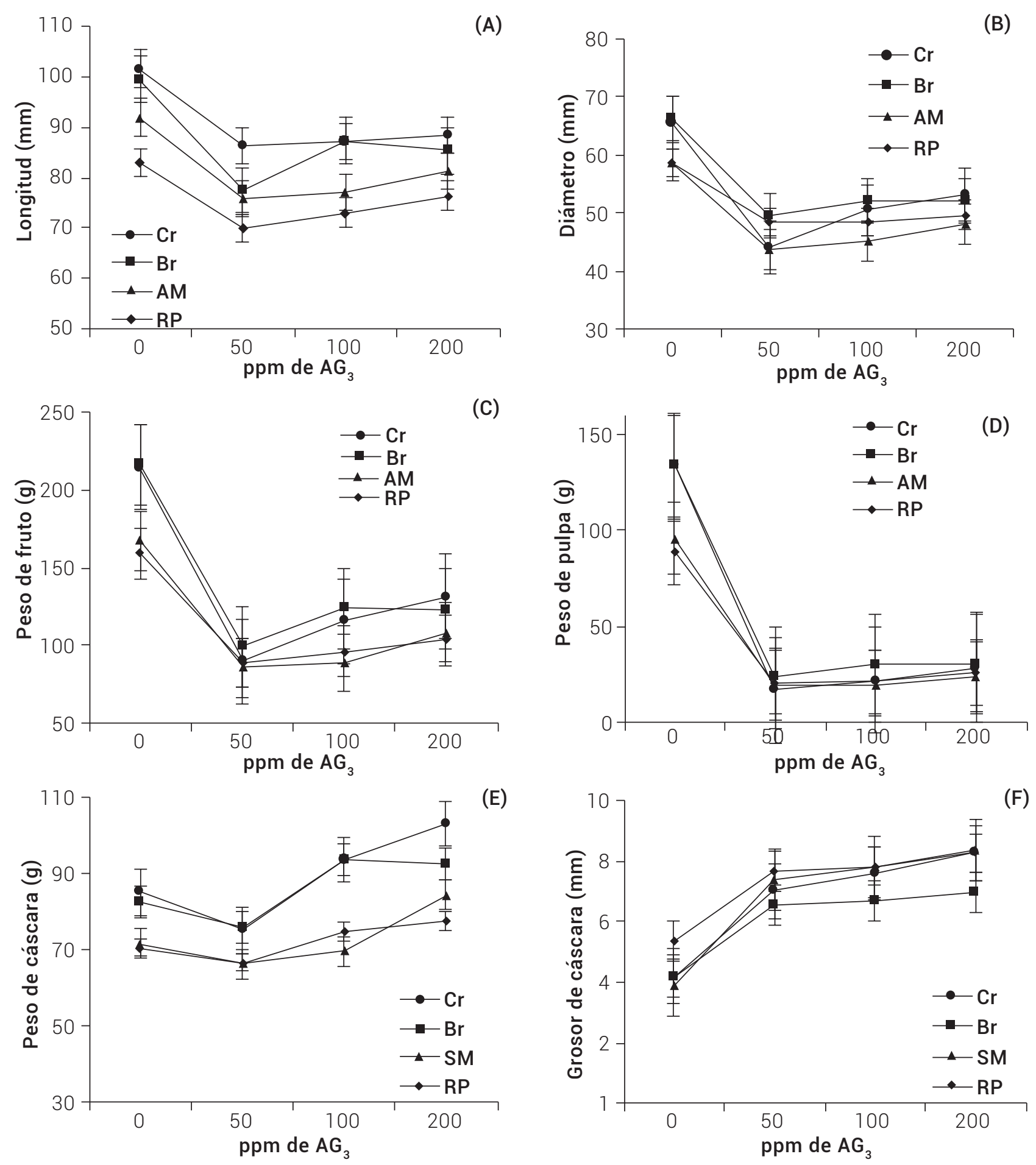

Figura 1. Características de calidad de tunas a diferentes concentraciones de ácido giberélico $\left(\mathrm{AG}_{3}\right)$ en cuatro variedades de nopal (Cr. Cristalina; Br. Burrona; AM: Amarilla Montesa y RP. Rojo Pelón). 
Los efectos del $\mathrm{AG}_{3}$ se han estudiado en 0 . ficus-indica (De la Barrera y Nobel, 2004; Díaz y Gil, 1978; Gil et al., 1977; Gil y Espinoza, 1980; Kaaniche-Elloumi et al., 2015), Opuntia amyclaea (Mejía y Cantwell, 2003; Ortiz et al., 1991) y Opuntia megacantha (Corrales y Hernández, 2005) en los que se aplicó $\mathrm{AG}_{3}$ solo o combinado con otros reguladores de crecimiento sobre flores emasculadas o en la antesis, logrando inhibir la formación de semillas total o parcialmente, pero con baja calidad de fruto, afectada principalmente por el mayor grosor de la cáscara, poca pulpa y frutos más pequeños que los testigos.

En comparación con los frutos normales, los tratados con $\mathrm{AG}_{3}$ presentaron una ligera disminución en los sólidos solubles totales en la pulpa y en la cáscara (Figuras 2C y D). Cristalina mostró una tendencia a incrementarlos conforme aumentó la dosis, pero no se encontraron diferencias significativas con respecto al testigo; Burrona presentó valores menores y estables en frutos tratados y no tratados; no se encontraron diferencias significativas entre tratamientos. Amarilla Montesa tuvo los valores más altos en ${ }^{\circ} \mathrm{Bx}$ de su cáscara cuando se aplicaron 50 y 100 ppm de $\mathrm{AG}_{3}$ (Figura 2D), con dulzor similar al de su pulpa (Figura 2E), lo que resulta importante si se desea consumir la cáscara.
Con la emasculación de las flores y la aplicación de $\mathrm{AG}_{3}$ se logró obtener frutos sin semillas normales, la pulpa sólo tuvo cubiertas seminales vacías (csv), sin embrión. Cristalina y Amarilla Montesa contrastan por su número de semillas y de csv en los frutos testigos y en los partenocárpicos, respectivamente (Cuadro 4); la primera tuvo el mayor número y la segunda el menor y no se encontraron diferencias significativas entre tratamientos. En Burrona la concentración de 200 ppm de $\mathrm{AG}_{3}$ disminuyó el número de cvs con respecto al número de semillas en el testigo, y Rojo Pelón presentó mayor número de csv en las tres concentraciones de $\mathrm{AG}_{3}$.

Los frutos partenocárpicos de las cuatro variedades tuvieron csv con un peso significativamente menor al peso de las semillas normales (Cuadro 5). Existe asociación entre el número de semillas normales y el tamaño y forma final de los frutos, ya que las semillas con embrión son de mayor tamaño y producen mayor cantidad de hormonas y pulpa (Gil et al., 1977). El tamaño final de la tuna depende del número de semillas fecundadas (Barbera et al., 1994), y la emasculación de flores evita la formación de semillas normales al impedir la formación de embriones (Gil et al., 1977).

Cuadro 4. Número de semillas en frutos normales y número de cubiertas seminales vacías en frutos partenocárpicos obtenidos con aplicación de tres dosis de $\mathrm{AG}_{3}$ en cuatro variedades de nopal tunero.

\begin{tabular}{lccccc}
\hline \multirow{2}{*}{${\mathrm{ppm} \mathrm{de} \mathrm{AG}_{3}}^{*}$ Tipo de fruto } & \multicolumn{4}{c}{ Variedad } \\
\cline { 3 - 6 } & Normal & Amarilla M. & Burrona & Cristalina & Rojo P. \\
\hline 0 & $252.0 \mathrm{a}$ & $327.0 \mathrm{a}$ & $374.7 \mathrm{a}$ & $245.1 \mathrm{~b}$ \\
50 & Partenocárpico & $274.1 \mathrm{a}$ & $288.3 \mathrm{~b}$ & $377.5 \mathrm{a}$ & $302.1 \mathrm{a}$ \\
100 & Partenocárpico & $279.6 \mathrm{a}$ & $305.3 \mathrm{ab}$ & $362.7 \mathrm{a}$ & $307.7 \mathrm{a}$ \\
200 & Partenocárpico & $271.0 \mathrm{a}$ & $284.8 \mathrm{~b}$ & $415.7 \mathrm{a}$ & $303.0 \mathrm{a}$ \\
DSH & & 43.1 & 37.7 & 65.5 & 54.6 \\
\hline
\end{tabular}

Medias con letras iguales en las columnas no son estadísticamente diferentes (Tukey, 0.05); DSH: diferencia significativa honesta.

Cuadro 5. Peso de semillas en frutos normales y peso de cubiertas seminales vacías ( $\mathrm{g}$ ) en frutos partenocárpicos obtenidos con aplicación de tres dosis de $\mathrm{AG}_{3}$ en cuatro variedades de nopal tunero.

\begin{tabular}{|c|c|c|c|c|c|}
\hline \multirow{2}{*}{ ppm de $\mathrm{AG}_{3}$} & \multirow{2}{*}{ Tipo de fruto } & \multicolumn{4}{|c|}{ Variedad } \\
\hline & & Amarilla M. & Burrona & Cristalina & Rojo P. \\
\hline 0 & Normal & $4.67 \mathrm{a}$ & $8.72 \mathrm{a}$ & $7.16 \mathrm{a}$ & $4.83 a$ \\
\hline 50 & Partenocárpico & $0.08 b$ & $0.07 \mathrm{~b}$ & $0.10 \mathrm{~b}$ & $0.11 \mathrm{~b}$ \\
\hline 100 & Partenocárpico & $0.08 \mathrm{~b}$ & $0.11 \mathrm{~b}$ & $0.11 \mathrm{~b}$ & $0.10 \mathrm{~b}$ \\
\hline 200 & Partenocárpico & $0.08 b$ & $0.11 \mathrm{~b}$ & $0.19 b$ & $0.14 b$ \\
\hline DSH & & 0.39 & 0.37 & 0.59 & 0.35 \\
\hline
\end{tabular}

Medias con letras iguales en las columnas no son estadísticamente diferentes (Tukey, 0.05); DSH: diferencia significativa honesta. 

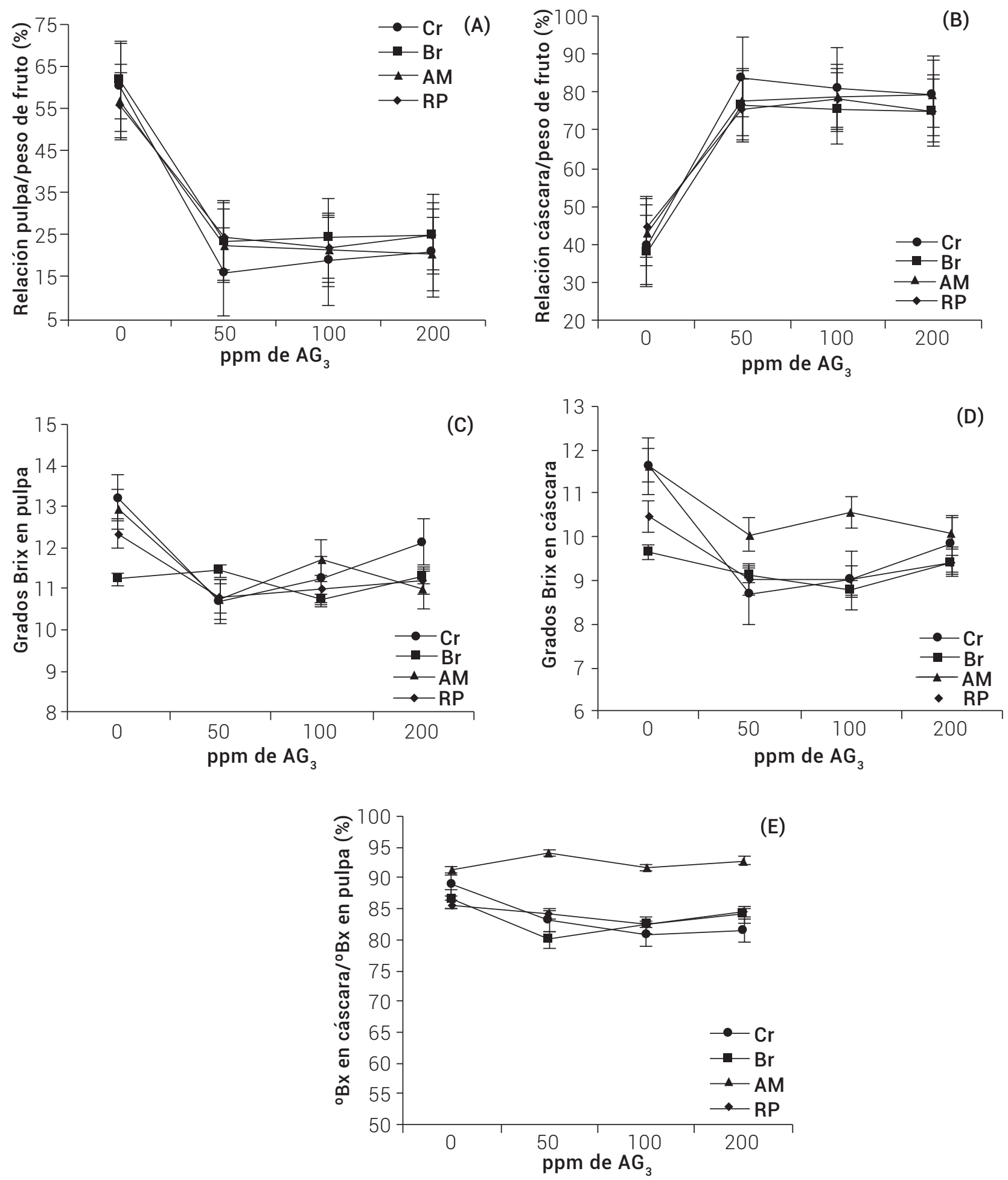

Figura 2. Características de calidad de tunas a diferentes concentraciones de ácido giberélico $\left(\mathrm{AG}_{3}\right)$ en cuatro variedades de nopal tunero (Cr. Cristalina; Br. Burrona; AM: Amarilla Montesa y RP. Rojo Pelón). 
Los resultados muestran que es posible producir frutos partenocárpicos con las cuatro variedades estudiadas y que es necesario explorar otras vías para mejorar la calidad del fruto; los mismos resultados indican que no es posible generalizar los efectos de la emasculación y aplicación de $\mathrm{AG}_{3}$ sobre la producción de pulpa y características de la cáscara porque la respuesta depende del genotipo, del ambiente y de la interacción entre genotipo y ambiente, y la emasculación de la flor y la aplicación de $\mathrm{AG}_{3}$ solamente son dos de los múltiples factores que afectan el resultado final.

\section{CONCLUSIONES}

La emasculación de flores en pre-antesis y la aplicación de 50, 100 y 200 ppm de $\mathrm{AG}_{3}$ indujeron la formación de frutos partenocárpicos en las variedades Cristalina (Opuntia albicarpa Sheinvar), Burrona (Opuntia albicarpa Sheinvar), Amarilla Montesa (Opuntia megacantha Salm-Dick) y Rojo Pelón (Opuntia ficus-indica L.). Los frutos partenocárpicos fueron de menor tamaño y peso que los frutos normales y tuvieron una relación peso de cáscara/peso de fruto más alta. Considerando que en la cáscara de los frutos partenocárpicos se encontraron grados Brix similares a los de su pulpa, para mejorar la calidad del fruto se puede considerar el desarrollo de tunas partenocárpicas con cáscara dulce comestible.

\section{AGRADECIMIENTO}

El primer autor agradece al Consejo Nacional de Ciencia y Tecnología el otorgamiento de la beca para realizar sus estudios de Maestría en Ciencias, durante los cuales se realizó la presente investigación.

\section{BIBLIOGRAFÍA}

Aguilar E. A., J. A. Reyes A. y J. R. Aguirre R. (2003) Caracterización de la semilla de 403 variantes de nopal (Opuntia spp.). In: Memoria del IX Congreso Nacional y VII Congreso Internacional sobre Conocimiento y Aprovechamiento del Nopal. G. Esparza, M. Salas, J. Mena y R. Valdez (eds.). UACh, UAZ e INIFAP. Zacatecas, México. pp:117-120.

ASERCA, Apoyos y Servicios a la Comercialización Agropecuaria (2011) Nopal y tuna, una mirada a su realidad actual. Claridades Agropecuarias 213:3-12
Bangerth F. and M. Schröder (1994) Strong synergistic effects of gibberellins with the synthetic cytokinin N-(2-chloro-4-pyridyl)$\mathrm{N}$-phenylurea on parthenocarpic fruit set and some other fruit characteristics of apple. Plant Growth Regulation 15:293-302.

Barbera G., P. Inglese and T. La Mantia (1994) Seed content and fruit characteristics in cactus pear (Opuntia ficus-indica Mill.). Scientia Horticulturae 58:161-165.

Corrales G. J. y J. L. Hernández S. (2005) Cambios en la calidad postcosecha de variedades de tuna con y sin semilla. Revista Fitotecnia Mexicana 28:9-16.

De la Barrera E. and P. S. Nobel (2004) Carbon and water relations for developing fruits of Opuntia ficus-indica (L.) Miller, including effects of drought and gibberellic acid. Journal of Experimental Botany 55:719-729.

Díaz Z. F. y G. Gil S. (1978) Efectividad de diversas dosis y métodos de aplicación del ácido giberélico en la inducción de partenocarpia y en el crecimiento del fruto de tuna (Opuntia ficus-indica Mill) Ciencia e Investigación Agraria 5:109-117.

ElBehi A. W., F. Orlandi, T. Bonofiglio, B. Romano, M. Fornaciari, P. Inglese, G. Sortino and G. Liguori (2015) Pollen morphology and reproductive performances in Opuntia ficus-indica (L.) Mill. Acta Horticulturae 1067:217-223.

Gallegos V. C. y C. Mondragón-Jacobo (2011) Cultivares Selectos de Tuna de México al Mundo. Servicio Nacional de Inspección y Certificación de Semillas, Secretaria de Agricultura, Ganadería, Desarrollo Rural, Pesca y Alimentación y Universidad Autónoma Chapingo. Chapingo, Edo. de México. 159 p.

Gil S. G. y A. Espinoza R. (1980) Desarrollo de frutos de tuna (Opuntia ficus-indica Mill.) con aplicación prefloral de giberelina y auxina. Ciencia e Investigación Agraria 7:141-147.

Gil G. F., M. Morales y A. Momberg (1977) Cuaja y desarrollo del fruto de tuna (Opuntia ficus-indica Mill) y su relación con polinización y con los ácidos giberélico y cloroetilfosfónico. Ciencia e Investigación Agraria 4:163-169.

Kaaniche-Elloumi N., E. Jedidi, K. B. Mahmoud, A. Chakroun and A. Jemmali (2015) Gibberellic acid application and its incidence on in vitro somatic embryogenesis and fruit parthenocarpy in an apomictic cactus pear (Opuntia ficus-indica (L.) Mill.) clone. Acta Horticulturae 1067:225-230.

Mejía A. and M. Cantwell (2003) Prickly pear fruit development and quality in relation to gibberellic acid applications to intact and emasculated flower buds. Journal of Professional Association for Cactus Development 6:72-85.

Ortiz H. Y. D., F. Barrientos P., M. T. Colinas L. y A. Martínez G. (1991) Ácido giberélico, auxinas y sus efectos sobre el fruto de nopal tunero. Agrociencia Serie Fitociencia 2:17-32.

Reyes-Agüero. J. A., J. R. Aguirre R., F. Carlín C. y A. González D. (2009) Catálogo de las Principales Variantes Silvestres y Cultivadas de Opuntia en la Altiplanicie Meridional de México. Universidad Autónoma de San Luis Potosí, Instituto de Investigación de Zonas Desérticas. San Luis Potosí, SLP. México. 350 p.

Sáenz H. C. (2004) Compuestos funcionales y alimentos derivados de Opuntia spp. In: El Nopal. Tópicos de actualidad. G. Esparza F. R. D. Valdés C. y S. J. Méndez G. (eds.). Universidad Autónoma Chapingo-Colegio de Postgraduados. Chapingo, Edo. de México. pp:211-222.

Weiss J., A. Nerd and Y. Mizrahi (1993) Vegetative parthenocarpy in the cactus pear Opuntia ficus-indica (L.) Mill. Annals of Botany $72: 521-526$. 